\title{
RESÍDUOS DA INDÚSTRIA PROCESSADORA DE POLPAS DE FRUTAS: CAPACIDADE ANTIOXIDANTE E FATORES ANTINUTRICIONAIS
}

\author{
Quesia Santos Amorim Martins* \\ Hanna Elísia Araújo de Barros** \\ Sandra Lúcia da Cunha e Silva*** \\ Simone Andrade Gualberto**** \\ Marcondes Viana da Silva*****
}

RESUMO: A grande extensão territorial do Brasil, aliada às condições adequadas de clima e do solo, permite que o país se destaque como o terceiro maior produtor mundial de frutas. Entretanto, constata-se que durante o processamento de polpas, sucos e outros derivados de frutas são gerados toneladas de resíduos, os quais são na maioria das vezes descartados de maneira inadequada, acarretando poluição ambiental. Sendo assim, objetivou-se com o presente estudo investigar a capacidade antioxidante e a presença de fatores antinutricionais em farinhas elaboradas a partir de resíduos de polpas de abacaxi, acerola, cajá, manga e maracujá, visando agregar valor comercial e mitigar seus impactos sobre o meio ambiente. Avaliou-se a capacidade antioxidante utilizando os ensaios da co-oxidação do $\beta$-caroteno e ácido linoleico e a degradação da 2-desoxirribose (2-DR). Dentre os fatores antinutricionais, investigou-se as determinações de oxalatos, nitratos, fitatos, atividade hemaglutinante (AHE) e saponinas totais. As farinhas de manga e maracujá destacaram-se com expressiva capacidade antioxidante. Todas as farinhas apresentaram baixos teores de fatores antinutricionais. Constata-se a partir desses resultados que há um amplo campo de possibilidades de investigação, relacionados com o aproveitamento integral de frutas tropicais.

PALAVRAS-CHAVE: Antinutrientes; Farinhas; Fitoquímicos bioativos; Reaproveitamento.

Mestre em Ciências Ambientais pela Universidade Estadual do Sudoeste da Bahia (UESB), Brasil.

** Mestranda em Engenharia e Ciência de Alimentos. Universidade Estadual do Sudoeste da Bahia (UESB), Brasil.

*** Docente Pleno da Universidade Estadual do Sudoeste da Bahia (UESB),Campus de Itapetinga, Brasil.

***** Docente Titular da Universidade Estadual do Sudoeste da Bahia (UESB). Doutorado em Ciências Farmacêuticas pela Universidade Federal de Minas Gerais, Brasil.

****** Docente Pleno da Universidade Estadual do Sudoeste da Bahia (UESB). Programas de Pós-Graduação em Engenharia e Ciência de Alimentos e Ciências Ambientais, Brasil. 


\title{
WASTES FROM FRUIT PULP PROCESSING INDUSTRY: ANTIOXI- DANT CAPACIT Y AND ANTI-NUTRITION FACTORS
}

\begin{abstract}
Brazil's great territorial extension and its climate and soil conditions make the country the third fruit producer worldwide. However, during the processing of pulp, juices and other fruit derivates, tons of wastes are produced which are normally discarded inadequately, with consequent environmental pollution. Current study investigates the antioxidant capacity and anti-nutrition factors in flour prepared from the wastes of the pulp of pineapple, acerola, cajá, mangoes and passionflower to aggregate commercial value and decrease impacts on the environment. Antioxidant capacity was assessed by assays involving co-oxidation of $\beta$-carotene and linoleic acid and the degradation of 2-desoxyrribose (2-DR). The amounts of oxalates, nitrates, phytates, hemaglutinant activity (HGA) and total saponins were investigated within the context of anti-nutrition factors. Mango and passionflower flours were highlighted for their antioxidant capacity. All types of flour had low rates of anti-nutritional factors. Results reveal a broad investigation field related to the integral reuse of tropical fruits.
\end{abstract}

KEY WORDS: Anti-nutrients; Phytochemical bioactive agents; Reuse.

\section{INTRODUÇÃO}

A fruticultura no Brasil encontra-se em expansão, além da vasta abundância de espécies produzidas nas diversas regiões, a modernização da agricultura, bem como as formas de apresentação e de industrialização colocam as frutas em destaque no agronegócio nacional (ANUÁRIO BRASILEIRO DA FRUTICULTUTRA, 2015).

Embora o consumo de frutas no Brasil (33 kg por habitante ao ano) seja bem inferior ao recomendado pela Organização Mundial de Saúde (100 kg por habitante ao ano), têm-se observado nas últimas décadas um aumento considerável na ingestão de frutas e hortaliças. Esse aumento é resultante da crescente preocupação do consumidor quanto à saúde e alimentação, aliado à melhoria na renda e maior qualidade das frutas. No entanto, a perecibilidade desses produtos corresponde a uma das principais barreiras para sua comercialização, principalmente no que se refere à exportação para longas distâncias (NEUTZLING et al., 2009; ANUÁRIO BRASILEIRO DA FRUTICULTUTRA, 2015). 
Uma das principais alternativas empregadas na busca pelo aproveitamento e conservação de frutas durante a safra é a fabricação de polpas de frutas congeladas, as quais possibilitam o armazenamento dos frutos, que poderão ser utilizados fora da sua época. O mercado de polpas no Brasil é promissor, pois a procura por produtos de fácil e rápido preparo é cada vez maior. Contudo, o aumento deste processamento gera resíduos que levam à preocupação quanto à sua forma de descarte, uma vez que os mesmos são potenciais poluidores por apresentarem, em sua maioria, elevado valor orgânico, por fornecerem nutrientes para microrganismos e, também, devido às perdas de biomassa e energia (ABUD; NARAIN, 2009; DENG et al., 2012).

Sendo assim, estudos que visem o aproveitamento desses resíduos são de grande importância, haja vista que podem contribuir para minimizar o seu impacto ambiental. Ademais, apresentam potencial nutricional e econômico. A elaboração de farinhas a partir de resíduos de frutas corresponde a uma alternativa viável de reaproveitamento, uma vez que estas podem ser utilizadas como ingredientes no preparo dos mais diversos produtos (biscoitos, bolos, pães, doces, dentre outros). Além disso, podem atuar como fonte enriquecedora de nutrientes (ZANATTA et al., 2010).

Diversos estudos explorando a utilização de resíduos industriais oriundos do processamento de alimentos (inclusive transformação de resíduos de frutas em farinhas) têm sido realizados, destacando-se a busca por substâncias naturais bioativas, principalmente os antioxidantes naturais, uma vez que estes são capazes de prevenir ou reduzir os danos do estresse oxidativo, como envelhecimento precoce e desenvolvimento de diversas doenças (OLIVEIRA et al., 2016; FREITAS et al., 2017).

Entretanto, observa-se que existe na literatura uma lacuna no que se refere ao estudo dos fatores antinutricionais presentes nesses resíduos. Tais estudos são de relevante importância, pois fornecem informações sobre a segurança na utilização desses produtos.

Como o próprio nome sugere, os fatores antinutricionais são substâncias que têm a capacidade de reduzir o valor nutritivo de um alimento, podendo atuar de diferentes maneiras no organismo, seja interferindo na digestibilidade do alimento, na sua absorção ou na utilização dos nutrientes presentes. Esses compostos, quando ingeridos em elevadas concentrações podem causar malefícios à saúde e até mesmo levar à morte (SANTOS, 2006; BENEVIDES et al., 2011).

Pelo exposto, objetivou-se com esse trabalho investigar a capacidade an- 
tioxidante e a presença de fatores antinutricionais em farinhas produzidas a partir dos resíduos de abacaxi, acerola, cajá, manga, e maracujá, obtidos de uma empresa processadora de polpas de frutas congeladas, situada na cidade de Vitória da Conquista, Bahia.

\section{MATERIAL E MÉTODOS}

\subsection{MATÉRIA-PRIMA}

Os resíduos das frutas foram doados por uma empresa processadora de polpas de frutas congeladas, situada na cidade de Vitória da Conquista (BA). Os resíduos foram coletados em três lotes, congelados $\left(-18 \pm 2{ }^{\circ} \mathrm{C}\right)$, que foram acondicionados em refrigerador à temperatura de $10 \pm 2{ }^{\circ} \mathrm{C}$ para descongelamento durante 24 horas. Posteriormente as amostras foram submetidas ao processo de desidratação em estufa com circulação e renovação de ar (Solab Cientifica, SL 102), à temperatura de $50 \pm 2{ }^{\circ} \mathrm{C}$, até atingir umidade inferior a $10 \%$. Posteriormente, foram colocadas em dessecador por duas horas.

Para obtenção das farinhas triturou-se os resíduos desidratados em moinho de facas (Willye, Marconi, Brasil). Para a padronização granulométrica das farinhas utilizou-se peneira com malha de 80 mesh. Visando evitar a degradação de compostos as farinhas foram armazenadas em temperatura de $-18 \pm 2{ }^{\circ} \mathrm{C}$, até as análises.

\subsection{OBTENÇÃO DOS EXTRATOS HIDROETANÓLICOS}

Os extratos hidroetanólicos (80:20 v. $\left.\mathrm{v}^{1}\right)$ foram obtidos de acordo com o procedimento proposto por Zhao e Hall (2008), com adaptações. Em um Becker foram adicionados $3 \mathrm{~g}$ da farinha do resíduo e $15 \mathrm{~mL}$ da solução extratora hidroetanólica. A mistura foi imersa em banho ultrassônico (UltraCleaner, USC-1400, Unique, Brasil), durante 25 minutos à temperatura ambiente. Posteriormente, o conteúdo foi centrifugado (Centrífuga Universal 320 R) a 8981 RCF - Força Centrífuga Relativa, por 5 minutos, e o sobrenadante foi recolhido. Em seguida, a farinha do resíduo foi submetida a mais duas reextrações sucessivas. Os extratos foram concentrados em 
evaporador rotativo (Fisaton, modelo 802, Brasil) com a temperatura da água a 50 ${ }^{\circ} \mathrm{C} \pm 1{ }^{\circ} \mathrm{C}$, após a evaporação do solvente foram armazenados em frasco de vidro âmbar ao abrigo da luz a $-4{ }^{\circ} \mathrm{C} \pm 2{ }^{\circ} \mathrm{C}$, onde permaneceram até o momento das análises de atividade antioxidante.

\subsection{AVALIAÇÃO DA CAPACIDADE OXIDANTE}

\subsubsection{Co-oxidação do Beta-caroteno e Ácido linoléico}

Adotou-se a metodologia descrita por Marco (1968), modificada por Emmons e Peterson (1999). Esse ensaio espectrofotométrico fundamenta-se na perda da coloração amarela do $\beta$-caroteno, provocada pela reação com os radicais formados da oxidação do ácido linoleico pela aeração do meio. A reação é acompanhada por espectrofotometria no visível em comprimento de onda de $470 \mathrm{~nm}$. A atividade antioxidante foi calculada como percentual de inibição, relativa ao controle, sendo utilizada a equação: \% de inibição $=100$ x DTc - DTa/DTc, onde DTc = Taxa de degradação do controle (Absorbância final do controle menos a absorbância inicial do controle) e DTa = Taxa de degradação da amostra (Absorbância final da amostra menos a absorbância inicial da amostra).

\subsubsection{Degradação Oxidativa da 2-desoxi-D-ribose (2DR)}

Determinou-se a capacidade antioxidante dos extratos pelo método da degradação oxidativa da 2-desoxi-D-ribose, proposto por Aruoma (1994). O ensaio consiste na degradação oxidativa da 2-desoxi-D-ribose (2-DR) pelo radical hidroxil, formado pela reação de Fento. A reação inicia-se com a redução do íon ( $\mathrm{Fe}^{3+}$ - EDTA) pelo ascorbato, gerando o complexo ( $\mathrm{Fe}^{2+}$. EDTA). Este reage com $\mathrm{H}_{2} \mathrm{O}_{2}$ formando o radical hidroxil, o qual provoca a degradação oxidativa da 2-DR, resultando na formação do malonaldeído (MAD) e dentre outros produtos secundários, o qual reage em meio ácido com o ácido 2-tiobarbitúrico (TBA) na proporção estequiométrica de (2:1) formando um éster com absorção máxima a $532 \mathrm{mn}$. Calculou-se a \% de inibição da formação do radical hidroxil de acordo com a Equação 1. 


\section{$\%$ deInibição}

$=\frac{\text { Abs. controle }- \text { Abs. amostra }}{\text { Abs. docontrole }} .100$

Eq. 1

\subsection{AVALIAÇÃO DOS FATORES ANTINUTRICIONAIS}

\subsubsection{Determinação do Teor de Ácido Oxálico}

Determinou-se o ácido oxálico segundo a metodologia proposta por Loures e Jokl (1990). O método consiste na extração do ácido oxálico sob aquecimento, com ácido clorídrico a $6 \mathrm{~N}$. Titulou-se o precipitado resultante com solução de permanganato de potássio $0,01 \mathrm{M}$. Os resultados foram expressos em $\mathrm{mg} .100 \mathrm{~g}^{-1} \mathrm{de}$ amostra.

\subsubsection{Determinação do Teor de Ácido Fítico}

Para a determinação do ácido fítico adotou-se o a metodologia proposta por Ruiz de Lope et al. (1982). O método fundamenta-se na determinação complexométrica do $\mathrm{Fe}^{3+}$ com EDTA, utilizando o ácido sulfosalicílico como indicador. Determinou-se a percentagem de ácido fítico na amostra utilizando-se a equação 2. Os resultados foram expressos em $\mathrm{mg}^{-1} \mathrm{~g}^{-1}$ de peso seca.

$$
\text { Ácido fítico }(\%)=\frac{0,66(10-V)}{P}
$$

Onde:

$\mathrm{V}=$ volume da solução de EDTA em mililitros;

$\mathrm{P}=$ peso da amostra em gramas;

Utilizou-se a razão atômica (4:6 Ferro/Fósforo $=0,66$ ). 


\subsubsection{Determinação de Nitratos}

Adotou-se a metodologia proposta por Cataldo et al. (1975). O ensaio fundamenta-se na complexação do ácido salicílico pelo íon nitrato sendo as absorbâncias medidas em espectrofotômetro (Shimadzu UV mini-1240) a $550 \mathrm{~nm}$. Para quantificação do nitrato foram construídas curvas analíticas, utilizando como padrão analítico uma solução de nitrato de potássio, variando as concentrações de 1 a $10 \mathrm{mg} \cdot \mathrm{mL}^{-1}$. Os resultados foram expressos em mg.100 g-1.

\subsubsection{Determinação Espectrofotométrica de Saponinas Totais}

Empregou-se o procedimento proposto por Vigo et al. (2003), utilizandose como reagente cromogênico cloreto de cobalto em meio ácido. Esse ensaio espectrofotométrico é realizado no comprimento de onda de $284 \mathrm{~nm}$. Para quantificação das saponinas totais foram construídas curvas analíticas utilizando solução de saponina nas concentrações variando de 0,08 a $0,32 \mathrm{mg} \cdot \mathrm{mL}^{-1}$. Os resultados foram expressos em $\mathrm{g} \cdot 100 \mathrm{~g}^{-1}$.

\subsubsection{Atividade Hemaglutinante}

Utilizou-se metodologia proposta por Figueroa e Lajolo (1997), o ensaio consiste na extração da hemaglutinina das farinhas em solução de $\mathrm{NaCl}$, agitandose por três horas, à temperatura ambiente e filtrando-se o extrato. A estimativa da atividade aglutinante foi realizada em placa de microtitulação, adicionando-se suspensão de eritrócitos (sangue humano $\mathrm{A}^{+}$) com o extrato das farinhas, em uma série de diluições, deixando em repouso por 1 hora, à temperatura ambiente, com verificação, por meio da leitura visual, da presença ou não de aglutinação de células sanguíneas. Como controle negativo foi utilizado apenas suspensão de eritrócitos, e como controle positivo foi utilizado extrato de feijão preto. 


\subsection{ANÁLISE ESTATÍSTICA}

Utilizou-se um delineamento de experimento inteiramente casualizado (DIC), com três repetições. Os resultados obtidos de três determinações foram submetidos à análise de variância (ANOVA) e as médias foram comparadas utilizando o teste de Tukey a 5\% de significância $(\mathrm{p}<0,05)$ por meio do software Assistência Estatística ASSISTAT, versão 7.7 (SILVA, 2016).

\section{RESULTADOS E DISCUSSÃO}

\subsection{AVALIAÇÃO DA CAPACIDADE ANTIOXIDANTE}

Os resultados relativos à capacidade antioxidante pelos métodos de co-oxidação do $\beta$-caroteno e ácido linoleico e o da degradação da 2-desoxirribose estão apresentados na Tabela 1 .

Tabela 1. Avaliação da capacidade antioxidante pelos métodos co-oxidação do $\beta$-caroteno e ácido linoleico e degradação da 2-desoxirribose (2-DR) em farinhas de resíduos de abacaxi, acerola, cajá, manga e maracujá

CAPACIDADE ANTIOXIDANTE

FARINHAS

$\beta$-caroteno/ácido linoleico

2-DR

(\% inibição da oxidação)

(\% inibição da oxidação)

\begin{tabular}{lcc}
\hline Abacaxi & $52,53^{\mathrm{c}} \pm 4,1$ & $12,08^{\mathrm{cd}} \pm 1,61$ \\
Acerola & $30,30^{\mathrm{d}} \pm 5,0$ & $20,73^{\mathrm{c}} \pm 5,18$ \\
Cajá & $68,37^{\mathrm{b}} \pm 4,5$ & $5,65^{\mathrm{d}} \pm 2,22$ \\
Manga & $51,00^{\mathrm{c}} \pm 4,5$ & $69,17^{\mathrm{a}} \pm 4,15$ \\
Maracujá & $75,42^{\mathrm{a}} \pm 5,2$ & $39,09^{\mathrm{b}} \pm 5,64$ \\
\hline
\end{tabular}

Médias seguidas das mesmas letras na mesma coluna não diferem estatisticamente entre si pelo teste de Tukey $(\mathrm{p}<0,05)$.

Fonte: Dados da pesquisa. 
Hassimotto et al. (2005) propuseram uma classificação para a capacidade antioxidante pelo método de co-oxidação do betacaroteno como sendo alta quando o percentual de inibição de oxidação for maior que 70\%, intermediária quando estiver entre 40 e 70\%, e baixa quando o percentual de inibição da oxidação for menor que $40 \%$. Com base nessa classificação, encontrou-se nesse estudo elevada capacidade antioxidante para farinha do resíduo de maracujá (75,42\%). As farinhas dos resíduos de cajá, abacaxi e manga apresentaram capacidade antioxidante moderada, enquanto que a farinha de acerola apresentou atividade antioxidante baixa.

Bergamaschi et al. (2010), ao avaliarem a capacidade antioxidante de diversos resíduos vegetais, encontraram valores bem inferiores ao desse trabalho em cascas de maracujá: 5,38\% no extrato etanólico e 13,53\% no extrato aquoso. Esses diferentes valores podem estar relacionados a vários fatores, tais como as condições do ambiente onde o experimento foi realizado, a espécie do fruto, o local de origem da matéria-prima, a presença de sinergismo, entre outras (LAGOURI; BOSKOU, 1995).

Araújo (2014), por sua vez, ao avaliar a atividade de farinhas obtidas de cascas de manga da variedade Tommy Atkins, encontrou 51,75\% de inibição da oxidação, valor bem semelhante ao desse trabalho (51,0\%). Enquanto Rufino et al. (2010), ao avaliar a capacidade antioxidante de diversas frutas, encontraram 84,9\% de inibição da oxidação para cajá; Melo et al. (2008) verificaram menos de $20 \%$ da inibição da oxidação em extratos acetônicos e aquosos de polpas de abacaxi, acerola e manga.

Pelo método da desoxirribose, o maior percentual de atividade antioxidante foi encontrado para farinha do resíduo de manga (69,17\%), seguido da farinha de maracujá (39,09\%), as demais farinhas apresentaram baixa atividade antioxidante. $\mathrm{Na}$ literatura consultada até o momento, observou-se ausência de dados referentes à atividade antioxidante, por esse método, em resíduos de frutas. Barros (2012), ao avaliar a atividade antioxidante em vários frutos, encontrou em extratos aquosos de cajá capacidade antioxidante maior $(79,90 \%)$ que a encontrada nesse trabalho $(39,09 \%)$ para farinha do resíduo dessa fruta. O mesmo autor observou elevada atividade antioxidante em extratos aquosos de seriguela (92,6\%), sapoti $(92,2 \%)$, carambola $(92,2 \%)$, e mangaba $(76,0 \%)$. 


\subsection{AVALIAÇÃO DA PRESENÇA DE FATORES ANTINUTRICIONAIS}

Na Tabela 2 estão expostos os resultados obtidos na avaliação dos fatores antinutricionais nas farinhas estudadas.

Tabela 2. Resultados das análises de fatores antinutricionais das farinhas de resíduos de abacaxi, acerola, cajá, manga e maracujá

DETERMINAÇÃO DE FATORES ANTINUTRICIONAIS

\begin{tabular}{lccccc}
\cline { 2 - 6 } FARINHAS & Oxalato & Fitato & $\begin{array}{c}\text { Nitrato } \\
\left(\mathrm{mg}^{\prime} 100 \mathrm{~g}^{-1}\right)\end{array}$ & $\begin{array}{c}\text { Saponinas } \\
\left(\mathrm{g} .100 \mathrm{~g}^{-1}\right)\end{array}$ & $\begin{array}{c}\text { Atividade he- } \\
\text { maglutinante }\end{array}$ \\
\hline Abacaxi & $1,37^{\mathrm{cd}} \pm 4,10$ & $1,97^{\mathrm{a}} \pm 0,07$ & $3,93^{\mathrm{d}} \pm 0,09$ & $0,12^{\mathrm{a}} \pm 0,02$ & $\mathrm{nd}$ \\
Acerola & $13,50^{\mathrm{a}} \pm 5,00$ & $0,95^{\mathrm{b}} \pm 1,50$ & $0,51^{\mathrm{e}} \pm 0,05$ & $0,13^{\mathrm{a}} \pm 0,03$ & $\mathrm{nd}$ \\
Cajá & $5,60^{\mathrm{b}} \pm 4,50$ & $0,55^{\mathrm{c}} \pm 0,05$ & $7,05^{\mathrm{b}} \pm 0,04$ & $0,21^{\mathrm{a}} \pm 0,05$ & $\mathrm{nd}$ \\
Manga & $2,20^{\mathrm{c}} \pm 4,50$ & $0,38^{\mathrm{c}} \pm 0,05$ & $9,34^{\mathrm{a}} \pm 0,10$ & $0,30^{\mathrm{a}} \pm 0,02$ & $\mathrm{nd}$ \\
Maracujá & $1,20^{\mathrm{d}} \pm 5,20$ & $\begin{array}{c}1,24^{\mathrm{ab}} \pm \\
0,02\end{array}$ & $5,89^{\mathrm{c}} \pm 0,04$ & $0,17^{\mathrm{a}} \pm 0,02$ & nd \\
\hline
\end{tabular}

Médias seguidas das mesmas letras na mesma coluna não diferem estatisticamente entre si pelo teste de Tukey $(\mathrm{p}<0,05)$. nd = não detectado.

Fonte: Dados da pesquisa.

Os teores de oxalato encontrados em todas as farinhas não foram expressivos, o maior valor foi encontrado na farinha do resíduo de acerola (13,50 mg.100 $\left.\mathrm{g}^{-1}\right)$, seguido da farinha de cajá (5,6 mg.100 $\left.\mathrm{g}^{-1}\right)$. De acordo com Ogbadoyi et al. (2006), o oxalato apresenta baixo limiar de toxicidade, sendo que a dose mínima considerada letal para adultos é em torno de 5 g. Sousa et al. (2014) encontraram $12 \mathrm{mg} \cdot 100 \mathrm{~g}^{-1}$ de oxalato em farinha produzida a partir de resíduos de acerola, valor semelhante ao encontrado nesse trabalho. Contudo, Sena et al. (2014) encontraram $24,93 \mathrm{mg} .100 \mathrm{~g}^{-1}$ de oxalato em farinhas de manga e $23,75 \mathrm{mg} .100 \mathrm{~g}^{-1} \mathrm{em}$ farinhas de cajá, valores superiores ao desse trabalho. Na literatura consultada não foram encontrados dados relativos ao teor de oxalato em farinhas de resíduos de abacaxi e maracujá, tampouco na polpa desses frutos.

Em relação aos fitatos os teores mais elevados foram encontrados nas farinhas de abacaxi (1,97 g.100 g $\left.\mathrm{g}^{-1}\right)$, maracujá $\left(1,24 \mathrm{~g} .100 \mathrm{~g}^{-1}\right)$ e acerola $\left(0,95 \mathrm{~g} .100 \mathrm{~g}^{-1}\right)$. 
Segundo Davis (1981), a partir de $1000 \mathrm{mg} .100 \mathrm{~g}^{-1}$, os fitatos já podem diminuir a biodisponibilidade de minerais bi e trivalentes. O Regulamento Técnico para a Fixação de Identidade e Qualidade de Mistura à Base de Farelos de Cereais determinava $0,1 \mathrm{~g} .100 \mathrm{~g}^{-1}$ como sendo o limite máximo permitido de fitato. De acordo com esse regulamento todas as farinhas estudadas apresentaram alto teor de fitato. Contudo, a Resolução no 263 de 22 de setembro de 2005 (Regulamento Técnico para Produtos de Cereais, Amidos, Farinhas e Farelos) revogou a Resolução anterior e não mais determina limites para o ácido fítico, justificando que este é um componente natural de cereais, raízes, tubérculos e leguminosas (BRASIL, 2000; BRASIL, 2005; LEAL et al., 2010). Marques et al. (2013), ao avaliarem o teor de fitatos em farinhas produzidas a partir de sementes e bagaços de acerola, encontraram 0,23 g. $100 \mathrm{~g}^{-1}$ nas farinhas da semente e $0,18 \mathrm{~g} .100 \mathrm{~g}^{-1}$ nas farinhas do bagaço, valores menores que os encontrados nesse trabalho na farinha do resíduo dessa fruta. De acordo com Brune et al. (1992), o teor de fitatos em farinhas é muito variável, sendo maior em farinhas não refinadas e obtidas de farelos do que em farinhas refinadas, isto porque durante o processamento das farinhas refinadas as camadas externas dos grãos são retiradas, enquanto que nas demais o ácido fítico continua presente na camada externa do grão.

Os teores de nitrato encontrados foram baixos em todas as farinhas, inclusive na farinha de resíduo de manga, que apresentou o maior teor de nitrato $(9,34$ mg. $\left.100 \mathrm{~g}^{-1}\right)$. De acordo com a Organização Mundial de Saúde a ingestão diária aceitável de nitrato é de $5 \mathrm{mg} \cdot \mathrm{kg}^{-1}$ de peso corporal. Dessa forma, um adulto com $70 \mathrm{~kg}$ precisaria ingerir diariamente mais de $3,7 \mathrm{~kg}$ de farinha de resíduo de manga para ultrapassar esse limite aceitável. Marques et al. (2010) encontraram em farinhas de semente de acerola ( $0,08 \mathrm{~g} .100 \mathrm{~g}$ 1) e em farinhas do bagaço de acerola $(0,20 \mathrm{~g} .100$ $\left.\mathrm{g}^{-1}\right)$ valores de nitrato superiores ao da farinha de resíduos de acerola encontrados nesse trabalho $\left(0,51 \mathrm{mg} \cdot 100 \mathrm{~g}^{-1}\right)$. Pereira et al. (2003) verificaram em folhas de cenoura 649,50 mg. $100 \mathrm{~g}^{-1}$ de nitrato. O local de origem das matérias-primas pode ser um dos fatores responsáveis pelos teores tão baixos de nitrato encontrados em todas as farinhas, isso porque esses teores na amostra podem variar com a quantidade de nitrato disponível no solo e água.

Os teores de saponinas encontrados em todas as farinhas foram baixos e se 
assemelham com os resultados de outros trabalhos com diferentes frutos e resíduos. Marques et al. (2013) verificaram os seguintes teores de saponinas nas farinhas das sementes de acerola: $0,49 \mathrm{~g} .100 \mathrm{~g}^{-1}$, e na farinha do bagaço dessa fruta: 0,26 g.100 $\mathrm{g}^{-1}$. Naves et al. (2010) verificaram $0,35 \mathrm{~g} .100 \mathrm{~g}^{-1} \mathrm{em}$ farinhas de semente de abóbora e teores variando de 0,24-0,31 g.100 $\mathrm{g}^{-1}$, quando essa farinha foi submetida a diferentes tratamentos térmicos. Lima et al. (2008), ao avaliarem o teor de saponinas no fruto inteiro, na polpa, na casca e na semente de duas variedades de jabuticaba (Sabará e Paulista) verificaram na variedade Sabará os seguintes teores de saponinas: fruto inteiro, 0,68 g. $100 \mathrm{~g}^{-1}$; polpa, 0,67 g. $100 \mathrm{~g}^{-1}$; casca, $0,63 \mathrm{~g} .100^{-1}$; e sementes, 0,34 g. $100^{-1}$. Na variedade Paulista os teores encontrados foram: fruto inteiro, 0,62 g.100${ }^{1}$; polpa, 0,66g.100 g-1; casca, 0,78g.100 g $\mathrm{g}^{-1}$; e sementes, $0,35 \mathrm{~g} .100 \mathrm{~g}^{-1}$; em ambas as variedades os mais baixos níveis de saponinas foram encontrados nas sementes.

Quanto às hemaglutininas, não foi detectada atividade hemaglutinante em nenhuma das farinhas estudadas, o que corresponde a um resultado favorável para consumo, haja vista que as hemaglutininas podem provocar efeitos degenerativos nas membranas celulares, dificultar a ação de enzimas digestivas e assim afetar a absorção de nutrientes, bem como interferir na eficácia do transporte de oxigênio para o organismo devido à aglutinação de células sanguíneas (PEREIRA et al., 2008; NAVES et al., 2010).

As análises foram realizadas com sangue humano tipo $\mathrm{A}^{+}$, conforme recomendado pela metodologia, entretanto não se pode eliminar a possibilidade que as mesmas farinhas apresentem atividade hemaglutinante em indivíduos com sangue do tipo B. Isso porque as células sanguíneas encontradas em sangues humanos de tipo A e B possuem diferentes carboidratos em sua superfície, e como as hemaglutininas são glicoproteínas com propriedade de ligar-se especificamente a certos carboidratos, há possibilidade de divergência entre os resultados com esses diferentes tipos sanguíneos (FUDENBERG et al., 1980; SHARON; LIS, 2004). 


\section{CONSIDERAÇÕES FINAIS}

A partir dos resultados obtidos constata-se que as farinhas analisadas apresentam potencial para aproveitamento como ingrediente na indústria alimentícia e farmacêutica, considerando sua expressiva capacidade antioxidante além de apresentarem baixos teores de fatores antinutricionais.

Assim sendo, abre-se um amplo campo de investigação quanto às possibilidades de aproveitamento integral de frutas tropicais. Ademais, constitui uma alternativa viável para minimizar os impactos ambientais decorrentes do descarte inadequado dos resíduos da indústria processadora de polpas de frutas.

\section{AGRADECIMENTOS}

À Coordenação de Aperfeiçoamento de Pessoal de Nível Superior (CAPES), pela concessão da bolsa de mestrado.

\section{REFERÊNCIAS}

ABUD, A. K. S.; NARAIN, N. Incorporação da farinha de resíduo do processamento de polpa de fruta em biscoitos: uma alternativa de combate ao desperdício. Brazilian Journal of Food Technology, v. 12. p. 247-265, 2009.

ANUÁRIO BRASILEIRO DA FRUTICULTURA. Santa Cruz do Sul: Editora Gazeta Santa Cruz, 1004 p., 2015.

ARAÚJO, C. R.; BOTELHO, P. S.; SILVA, T. M. S.; MACIEL, M. I. S.; MELO, E. A. RYAN, S.; SMITH, R. E. Phenolic Compounds and Metals in Tommy Atkins Mango (Mangifera indica L) Peels. The Natural Products Journal, v. 4, p. 2-7, 2014.

ARUOMA, O. Deoxyribose assay for detecting hydroxyl radicals. Methods in Enzymology, v. 233, p. 57-66, 1994.

BARROS, J. A. C. Avaliação da atividade antioxidante e antiproliferativa do ex- 
trato aquoso de frutas tropicais. Dissertação (mestrado) - Programa de Pós-Graduação em Ciências da Saúde/Universidade Federal do Rio Grande do Norte, 74 p., 2012.

BENEVIDES, C. M. J.; SOUZA, M. V.; SOUZA, R. D. B.; LOPES, M. V. Fatores antinutricionais em alimentos: Revisão. Segurança Alimentar e Nutricional, v. 18, p. 67-79, 2011.

BERGAMASCHI, K. B.; MELO, P. S.; PRADO, A.; TIVERON, A. P.; OLDONI, T. L. C.; LACERDA, R. C. C.; BEZERRA, R. M. N.; MORENO, I.; ALENCAR, S. M. Antioxidant activity and phenolic composition of aqueous extracts of vegetable and agro-industrial residues. In: 16th World Congress of Food Science and Technology, 2012.

BRASIL. Resolução RDC $\mathbf{n}^{\mathbf{0}}$ 53, de 15 de junho de 2000. Regulamento Técnico de Identidade e Qualidade de Mistura à base de Farelo de Cereais. ANVISA - Agência Nacional de Vigilância Sanitária, 2000.

BRASIL. Resolução RDC no 263, de 22 de setembro de 2005. Regulamento técnico para produtos de cereais, amidos, farinhas e farelos. ANVISA - Agência Nacional de Vigilância Sanitária, 2005.

BRUNE, M.; ROSSANDER-HULTÉN, L.; HALLBERG, L.; GLEERUP, A.; SANDBERG, A. Iron absorption from bread in humans: inhibiting effects of cereal fiber, phytate and inositol phosphates with different numbers of phosphate groups. Journal of Nutrition, v. 122, p. 442-449, 1992.

BRUNINI, M. A.; DURIGAN, J. F.; OLIVEIRA, A. L. Avaliação das alterações em polpa de manga 'Tommy-atkins' congeladas. Revista Brasileira de Fruticultura, v. 24, p. 651-653, 2002.

CATALDO, D. A.; HAROON, M.; SCHRADER, L. E.; YOUNGS, V. L. Rapid Colorimetric Determination of Nitrate in Plant-Tissue by Nitration of Salicylic-acid. Comunication in Soil Science and Plant Analysis. v. 6, p. 671-680, 1975.

COSTA, D. A.; OLIVEIRA, G. A. L.; SOUSA, D. P.; FREITAS, R. M. Avaliação do potencial antioxidante in vitro do composto ciano-carvona. Revista de Ciências Farmacêuticas Básica e Aplicada, v. 23, p. 567-575, 2012. 
DAVIS, K. R. Proximate composition, phytic acid, and total phosphorus of selected breakfast cereals. Cereal Chemistry, v. 58, p. 347-350, 1981.

DENG, GUI-FANG.; SHEN, C.; XU, XIANG-RONG.; KUANG, RU-DAN.; GUO, YA-JUN.; ZENG, LI-SHAN.; GAO, LI-LI.; LIN, X.; XIE, JIE-FENG.; XIA, EN-QIN.; LI, S.; WU, S.; CHEN, F.; LING, WEN-HUA.; LI, HUA-BIN. Potential of Fruit Wastes as Natural Resources of Bioactive Compounds. International Journal of Molecular Sciences, v. 13, p. 8308-8323, 2012.

EMMONS, C. L.; PETERSON, D. M.; PAUL, G. L. Antioxidant capacity of oat (Avena sativa L.) extracts 2 . In vitro antioxidant activity and content of phenolic and tocol antioxidants. Journal of Agricultural and Food Chemistry, v. 47, p. 4894-4898, 1999.

FERREIRA, G. A. A. Ião nitrato em alfaces cultivadas em hortas. Trabalho final de conclusão de curso. Universidade Atlântica, 48 p., 2011.

FIGUEROA, M.; LAJOLO, F. M. Effect of chemical modification of Phaseoulus vulgares lectins on their biological properties. Journal of Agricultural and Food Chemistry, v. 45, p. 639-643, 1997.

FREITAS, E. C.; BARROS, H. E. A.; SANTOS, I. A.; MIRANDA, A. S.; SANTANA, R. F.; SILVA, M. V. Constituintes Fenólicos e Screening da Capacidade Antioxidante de Coprodutos Desidratados de Theobroma grandiflorum. Revista Virtual Química, v. 9, p. 2193-2203. 2017.

FUDENBERG, H. H.; STITES, D. P.; CALDWELL, J. L.; WELLS, J. V. Imunologia Básica e Clínica. $2^{\mathrm{a}}$ ed. Rio de Janeiro: Guanabara Koogan, 1980.

HASSIMOTTO, N. M. A.; GENOVESE, I. S.; LAJOLO, F. M. Antioxidant activity of dietary fruits, vegetables, and commercial frozen fruit pulps. Journal of Agricultural and Food Chemistry, v. 53, p. 2928-2935, 2005.

LAGOURI, V.; BOSKOU, D. Screening for antioxidant activity of essential oils obtained from spices. Developments in Food Science, v. 37, p. 869-879, 1995.

LEAL, A. S.; GONÇALVES, C. G.; VIEIRA, I. F. R.; CUNHA, M. R. R.; GOMES, T. C. B.; 
MARQUES, F. R. Avaliação da concentração de minerais e dos fatores antinutricionais fitato e oxalato em multimisturas da Região Metropolitana de Belo Horizonte/MG. Nutrire: Revista da Sociedade Brasileira de Alimentação e Nutrição, v. 35, p. 39-52, 2010.

LIMA, A. J. B.; CORREAA, A. D.; ALVES, A. P. C.; ABREU, C. M. P.; BARROS, A. M. D. Caracterização química do fruto jabuticaba (Myrciaria cauliflora Berg) e de suas frações. Archivos Latinoamericanos de Nutricion, v. 58, p. 416-421, 2008.

LOURES, A.; JOKL, L. Microtécnica para determinação de ácido oxálico em folhas e derivados. In: Encontro nacional de analistas de alimentos. Resumos: Curitiba: Instituto de Tecnologia do Paraná, p. 59, 1990.

MARQUES, A.; CHICAYBAM, G.; ARAÚJO, M. T.; MANHÃES, L. R. T.; SABAA-SRUR, A. U. Composição centesimal e de minerais de casca e polpa de manga (Mangifera indica cv. Tommy Atkins). Revista Brasileira de Fruticultura, v. 32, p. 1206-1210, 2010.

MARQUES, T. R.; CORRÊA, A. D.; LINO, J. B. R.; ABREU, C. M. P.; SIMÃO, A. A. Chemical constituents and technological functional properties of acerola (Malpighia emarginata DC.) waste flour. Food Science and Technology, v. 33, p. 526-531, 2013.

MELO, E. A.; MACIEL, M. I. S.; LIMA, V. L. A. G.; ARAÚJO, C. R. Teor de Fenólicos Totais e Capacidade Antioxidante de Polpas Congeladas de Frutas. Alimentos e Nutrição, v. 19, p. 62-72, 2008.

NAVES, L. P.; CORRÊA, A. D.; SANTOS, C. D.; ABREU, C. M. P.; Componentes antinutricionais e digestibilidade proteica em sementes de abóbora (Cucurbita maxima) submetidas a diferentes processamentos. Ciência e Tecnologia de Alimentos, v. 30, p. 180-184, 2010.

NEUTZLING, M. B.; ROMBALDI, A. J.; AZEVEDO, M. R.; HALLAL, P. C. Fatores associados ao consumo de frutas, legumes e verduras em adultos de uma cidade no Sul do Brasil. Caderno de Saúde Pública, v. 25, p. 2365-2374, 2009.

OGBADOYII, E. O.; MAKUN, H. A.; BAMIGBADE, R. O.; OYEWALE, A. O.; OLADIRAN, $\mathrm{J}$. A. The effect of processing and preservation methods on the oxalate levels of some 
Nigerian leafy vegetables. Biokemistri, v. 18, p. 121-125, 2006.

OLIVEIRA, J. B.; NEVES, J. V. G.; SILVA, M. V. Phytochemical analysis and antioxidant activity of the hydroethanolic extract of Passiflora edulis $\mathrm{f}$. flavicarpa residues. $\mathbf{B}$. CEPPA, v. 34, p. 75-84, 2016.

PEREIRA, G. I. S.; PEREIRA, R. G. F. A.; BARCELOS, M. F. P.; MORAIS, A. R. Avaliação química da folha de cenoura visando seu aproveitamento na alimentação humana. Ciência e Agrotecnologia, v. 27, p. 852-857, 2003.

PEREIRA, C. A.; CORRÊA, A. D.; SANTOS, C. D.; ABREU, C. M. P.; SOUSA, R. V.; MAGALHÃES, M. M. Hemaglutinina de folhas de mandioca (Manihot esculenta Crantz): purificação parcial e toxicidade. Ciência e Agrotecnologia, v. 32, p. 900-907, 2008.

RUFINO, M. S. M.; ALVES, R. E.; BRITO, E. S.; PÉREZ-JIMÉNEZ, J.; SAURA-CALIXTO, F.; MANCINI-FILHO, J. Bioactive compounds and antioxidant capacities of 18 non-traditional tropical fruits from Brazil. Food Chemistry, v. 121, p. 996-1002, 2010.

RUIZ DE LOPE, C.; GARCÍA-VILLANOVA, R. J.; GARCÍA-VILLANOVA, R. Estudio del contenido en ácido fítico durante el processo de elaboración del pan blanco, pan integral y pan ázimo. Ars Pharmaceutica, v. 23, p. 437-442, 1982.

SANTOS, M. A. T. Efeito do cozimento sobre alguns fatores antinutricionais em fothas de brócolis, couve e couve-flor. Ciência Agrotécnica, v. 30, p. 294-301, 2006.

SENA, D. N.; ALMEIDA, M. M. B.; SOUSA, P. H. M.; GONZAGA, M. L. C.; SILVA, A. M. M. Avaliação dos teores de oxalato em farinha de resíduos de frutas tropicais. Revista Magistra, v. 26, p. 456-459, 2014.

SHARON, N.; LIS, H. History of lectins: from hemaglutinins to biological recognition molecules. Glycobiolog y, v. 14, p. 53-62, 2004.

SILVA, F. de A. S.; AZEVEDO, C. A. V. de. The Assistat Software Version 7.7. and it use in the analyses of experimental data. African Journal of Agricultural Research, v. 11, n. 39, p. 3733-3740, 2016. 
SOUSA, M. M. A.; SENA, D. N.; ALMEIDA, M. M. B.; SOUSA, P. H. M.; FIGUEREDO, R. W. Avaliação dos teores de oxalato em farinha de resíduos de acerola, graviola e tangerina. In: XX Congresso Brasileiro de Engenharia Química - Anais COBEQ, 2014.

VIGO, C. L. S.; NARITA, E.; MARQUES, L. C. Validação da metodologia de quantificação espectrofotométrica das saponinas de Pfafia glomerata (Spreng) Pedersen - Amaranthaceae. Revista Brasileira de Farmacognosia, v. 13, p. 46-49, 2003.

ZANATTA, C. L.; SCHLABITZ, C.; ETHUR, E. M. Avaliação físico-química e microbiológica de farinhas obtidas a partir de vegetais não conformes à comercialização. Alimentos e Nutrição, v. 21, p. 459-468, 2010.

ZHAO, B.; HALL, C. A. Composition and antioxidant activity of raisin extracts obtained from various solvents. Food Chemistry, v. 108, p. 511-551, 2008.

Recebido em: 11/04/2016

Aceito em: 24/05/2018 